\title{
Cytotoxicity study on SH-SY5Y cells cultured at high glucose levels and treated with bupivacaine
}

\author{
YAWEN LI ${ }^{1,2}$, SHIYUAN XU ${ }^{1}$, QINGGUO ZHANG ${ }^{1}$, LE LI $^{1}$, LUYING LAI $^{1}$, \\ TING ZHENG ${ }^{1}$, JIAOLING SU ${ }^{2}$, NAIMEI YANG ${ }^{2}$ and YUANTAO $\mathrm{LI}^{2}$ \\ ${ }^{1}$ Department of Anesthesia, Zhujiang Hospital, Southern Medical University, Guangzhou, \\ Guangdong 510282; ${ }^{2}$ Department of Anesthesia, Shenzhen Maternal and Child Health Hospital, \\ Southern Medical University, Shenzhen, Guangdong 510282, P.R. China
}

Received April 4, 2013; Accepted November 6, 2013

DOI: $10.3892 / \mathrm{mmr} .2013 .1843$

\begin{abstract}
The aim of this study was to investigate susceptibility to the neurotoxicity of local anesthetic agents in a model of gestational diabetes mellitus (GDM). SH-SY5Y cells were cultured at different concentrations of glucose and subsequently treated with $1 \mathrm{mmol} / \mathrm{l}$ bupivacaine for $6 \mathrm{~h}$. Reactive oxygen species (ROS) production and apoptosis were assessed using flow cytometry in each group of cells. The MTT method was utilized to detect cell survival, and western blot analysis was used to examine changes in $78 \mathrm{kDa}$ glucose-regulated protein (GRP78) levels in neuronal cells. In all groups, levels of ROS production, cell survival and GRP78 expression were significantly different $(\mathrm{P}<0.01)$ following the addition of various concentrations of glucose and bupivacaine, as well as for the interaction between different concentrations of the anesthetic agents, demonstrating a statistically significant difference. In conclusion, the susceptibility of SH-SY5Y cells to the neurotoxicity of local anesthetic agents was enhanced in a model of GDM.
\end{abstract}

\section{Introduction}

Neurological complications occur in certain parturient females following intraspinal anesthesia. The incidence is increased in patients with gestational diabetes mellitus (GDM). Of all the severe neurological complications, spinal nerve dysfunction is the most common. The major manifestation is the enhanced susceptibility of patients with GDM to the neurotoxicity of local anesthetic agents (1). It has been demonstrated that following intraspinal anesthesia or analgesic therapy in patients

Correspondence to: Dr Shiyuan Xu, Department of Anesthesia, Zhujiang Hospital, Southern Medical University, 1838 Guangzhou Avenue North, Guangzhou, Guangdong 510282, P.R. China E-mail: shiyuan_xu@126.com

Key words: SH-SY5Y cells, reactive oxygen species, cell apoptosis, susceptibility to neurotoxicity of local anesthetic agents with diabetes, the risk of neural damage is significantly higher. Alternatively, it may cause the existing polyneuropathy to deteriorate. However, little literature and few retrospective studies regarding the susceptibility of patients with GDM to the neurotoxicity of local anesthetic agents are available. Disciplines associated with the ultrastructure, metabolism and electrophysiological damage of nerve tissue in patients with GDM following the use of local anesthetic agents, as well as studies regarding how to reduce such damages, are likely to be of clinical concern.

GDM refers to a glucose tolerance anomaly that first appears or is first detected during pregnancy (2). According to global statistics, the incidence of GDM is 3-5\% among all pregnant females and is more common in the intermediate and late stages of pregnancy (3). It has been reported that the incidence of GDM in China has risen to 7\% (4). GDM is a specific type of diabetes occurring in pregnant females and is different from type I and II diabetes, and from high blood sugar. The causes may be associated with higher levels of hormones that are antagonistic to insulin in the intermediate and late stages of pregnancy, including human placental lactogen, free cortisol, progestin and prolactin. Other factors may enhance the antagonism to insulin during pregnancy. A study revealed that endoplasmic reticulum (ER) stress may be associated with the metabolic disorder in diabetes (5). It is necessary for the internal environment of the ER to be stabilized in order for it to fulfill its basic functions. The ER has a powerful homeostasis system, which is constantly challenged by numerous factors that lead to an imbalanced homeostasis; a possible consequence is ER stress. Females with GDM are usually in a state of overnutrition $(3,6,7)$. Energy and nutrition surplus may cause ER stress (8), inhibit insulin, and eventually lead to stress on organelles and mitochondrial dysfunction. In response to this, reactive oxygen species (ROS) are produced. Enhanced levels of ROS in cells act as a positive feedback to ER stress, and further enhance ER stress. Therefore, chronic ER stress facilitates the toxic accumulation of ROS in cells, leading to apoptosis.

It has been experimentally demonstrated that local anesthetic agents trigger ER stress, and, in response, higher levels of intracellular ER stress are generated. Park et al (9) found that bupivacaine $(0.5 \mathrm{mM})$ induces the generation of high levels 
of ROS in Schwann cells, activating caspase 3 (a protease associated with apoptosis in cells) and, therefore, causing apoptosis. It may be inferred that the induction of apoptosis by bupivacaine proceeds via the increase in ROS levels. The presence of bupivacaine activates Schwann cells, leading to apoptosis through the production of ROS. Schwann cells, when damaged, block the conduction of nerve impulses, while minor damage to the myelin sheath or the Schwann cells may slow down the conduction. It was proposed by Park et al that bupivacaine serves as a trigger of apoptosis in Schwann cells by initiating ROS production. Zhou et al (10) demonstrated that ropivacaine was capable of increasing ROS production in SH-SY5Y cells via 5' adenosine monophosphate-activated protein kinase, leading to apoptosis.

ER stress effects changes in the expression of $78 \mathrm{kDa}$ glucose-regulated protein (GRP78), while severe, long-term ER stress may ultimately result in apoptosis (11-13). To date, the specific mechanism by which ER stress leads to the apoptosis of neuronal cells has yet to be elucidated.

In conclusion, two factors (GDM and the use of local anesthetic agents) promote ER stress and thus increase ROS production in cells, which leads to apoptosis. Furthermore, the pathophysiological mechanism as to how these two factors interact when intraspinal anesthesia is used in patients with GDM, leading to an increased susceptibility to the neurotoxicity of local anesthetic agents, has yet to be elucidated. However, few experiments have been conducted regarding this issue. The present study aimed to investigate the interaction between an in vitro cell model of GDM and local anesthetic agents. Experiments were performed to show the pathophysiological mechanisms of susceptibility to the neurotoxicity of local anesthetic agents in a model of GDM.

\section{Materials and methods}

Cells and reagents. The SH-SY5Y cell line was purchased from the Cell Resources Center, Shanghai Institute for Biological Sciences (Shanghai, China). The cells were cultured in $10 \mathrm{ml}$ Dulbecco's modified Eagle medium with Ham's F12 medium (DMEM/F12) containing $15 \%$ fetal bovine serum. DMEM/F12 culture medium and DMEM low-sugar medium were purchased from Gibco-BRL (Carlsbad, CA, USA).

Analysis of ROS production of cells incubated at high glucose levels. DMEM containing glucose at concentrations of 5.6, 6.1, $7.0,7.8,11.1$ and $13.3 \mathrm{mmol} / 1$, respectively, was prepared.

SH-SY5Y cells were seeded in two 6 -well plates at $5 \times 10^{5}$ cells/well. Control, positive, negative and blank wells were set up. Following incubation for one day, the glucose-containing culture medium was replaced for each well and incubated for a further two days. The fluorescent probe, 2'-7'-dichlorodihydrofluorescein diacetate (DCFH-DA), was diluted 1:1,000 with phosphate-buffered saline (PBS) to reach a final concentration of $10 \mu \mathrm{g} / \mathrm{l}$. The culture medium was removed and $100 \mu \mathrm{l}$ DCFH-DA was added to each well. Following incubation at $37^{\circ} \mathrm{C}$ for $20 \mathrm{~min}$, the cells were washed with PBS three times, to remove any DCFH-DA that failed to enter the cells. The cells were then re-suspended to prepare single cell suspensions. Flow cytometry (FCM) detection was performed with a FACSCalibur flow cytometer (BD Biosciences, New York, NY,
USA) to measure the intensity of fluorescence in the cells. The excitation wavelength was $488 \mathrm{~nm}$ and the emission wavelength was $525 \mathrm{~nm}$.

Analysis of ROS levels in cells treated with bupivacaine. DMEM containing glucose at concentrations of 5.6, 6.1, 7.0, 7.8, 11.1 and $13.3 \mathrm{mmol} / 1$, respectively, was prepared. SH-SY5Y cells were seeded into two six-well plates at $5 \times 10^{5} /$ well. Control, positive, negative and blank wells were set up. After one day, the culture medium was replaced for each well and the cells were incubated overnight. The cells were then treated with $1 \mathrm{mmol} / \mathrm{l}$ bupivacaine for 4-6 h. The detection of ROS was then performed in accordance with the aforementioned method.

MTT assay of cell survival following incubation at high glucose levels. The cells were seeded into a 96-well plate, which was divided into six sections. To each well, $100 \mu 1$ glucose solution was added. Sterilized PBS was used to fill the wells to the edge. A total of $20 \mu \mathrm{l}$ MTT solution $(5 \mathrm{mg} / \mathrm{ml})$ was added to each well, and the cells were incubated at $37^{\circ} \mathrm{C}$. Four hours later, the supernatant was discarded and $150 \mu \mathrm{l}$ dimethylsulfoxide was added into each well. The cells were agitated until all the purple crystals were dissolved.

MTT assay of the impact of local anesthetic agents on the survival of cells incubated at high glucose levels. The cells were seeded into a 96-well plate, which was divided into six sections. To each well, $100 \mu 1$ glucose medium was added. Sterilized PBS was used to fill the wells to the edge. The glucose-containing medium was then replaced for each group, and the cells were incubated for one more day. The medium was subsequently removed, $100 \mu$ l bupivacaine solution $(1 \mathrm{mmol} / \mathrm{l})$ was added to each well and the plate containing the cells was incubated at $37^{\circ} \mathrm{C}$ under $5 \% \mathrm{CO}_{2}$ for $6 \mathrm{~h}$.

FCM detection of apoptosis of cells incubated at high glucose levels. The concentration was adjusted to $5 \times 10^{5} / \mathrm{ml}$ by counting the cells. The cells were then seeded in a 24-well plate and $500 \mu \mathrm{l}$ glucose solution was added to each well. The plate was incubated at $37^{\circ} \mathrm{C}$ and under $5 \% \mathrm{CO}_{2}$ for one day. The glucose-containing medium of each section was replaced and the cells were incubated for one more day. The media in the 24-well plate were removed and the cells were treated with $1 \mathrm{mmol} / \mathrm{l}$ bupivacaine for $6 \mathrm{~h}$. Annexin V-fluorescein isothiocyanate (FITC) $(5 \mu \mathrm{l})$ and trypsin $(5 \mu \mathrm{l})$ were added to each well, and the cells were incubated at room temperature for $5 \mathrm{~min}$ in the dark. The cells were then analyzed with flow cytometry using the FACSCalibur flow cytometer.

Western blot analysis of the expression levels of GRP78. The cell mixture was fully suspended by swirling and incubated on ice for $30 \mathrm{~min}$. The mixture was then centrifuged at $4^{\circ} \mathrm{C}$ for $20 \mathrm{~min}$, the supernatant was removed and the pellet was subjected to the electrophoresis experiment. Briefly, the protein was subjected to polyacrylamide gel electrophoresis (PAGE), and transferred to a polyvinylidene fluoride (PVDF) membrane. After blocking, the PVDF membrane was washed with TBST for 5 min three times, then incubated with rabbit anti-human GRP78 (Abcam, Cambridge, England) antibody 
overnight and mouse anti-rabbit secondary antibody (Abcam) for $1 \mathrm{~h}$, respectively. Lastly, the PVDF membrane was developed with ECL chemiluminescence

Statistical analysis. Data are expressed as the mean \pm standard deviation. Data analysis was performed with SPSS 13.0 statistical software (SPSS, Inc., Chicago, IL, USA). Cell survival, ROS content and apoptosis data were subjected to variance analysis by two-factor factorial design. Inter-group comparison was performed by the least significant difference test (homogeneity of variance) or Dunnett's T3 test (inhomogeneity of variance). $\mathrm{P}<0.05$ was used to indicate a statistically significant difference.

\section{Results}

Analysis of ROS production of cells. As shown in Fig. 1, the number of ROS was enhanced with increasing glucose concentration. There were statistically significant differences between the glucose and the glucose + bupivacaine groups at glucose concentrations of $5.56 \mathrm{mmol} / \mathrm{l}(\mathrm{P}<0.01), 7.8 \mathrm{mmol} / \mathrm{l}(\mathrm{P}<0.05)$, $11.1 \mathrm{mmol} / \mathrm{l}(\mathrm{P}<0.01)$ and $13.3 \mathrm{mmol} / \mathrm{l}(\mathrm{P}<0.05)$; however, no statistically significant differences were identified in the 6.1 and $7.0 \mathrm{mmol} / 1$ groups compared with the glucose + bupivacaine groups $(\mathrm{P}>0.05)$.

Cell survival assay. As shown in Fig. 2, the cell viability was assessed in an MTT assay. The cell viability was enhanced with increasing glucose concentration. There were statistically significant differences between the glucose and the glucose + bupivacaine groups at glucose concentrations of $5.56 \mathrm{mmol} / 1,6.1 \mathrm{mmol} / 1,7.0 \mathrm{mmol} / 1,7.8 \mathrm{mmol} / 1,11.1 \mathrm{mmol} / 1$ and $13.3 \mathrm{mmol} / \mathrm{l}(\mathrm{P}<0.001)$.

FCM detection of apoptosis. As shown in Fig. 3, apoptosis was investigated using FCM. The apoptosis rate was 0.014, 0.017, $0.026,0.029,0.047$ and 0.05 at glucose concentrations ranging between 5.56 and $13.3 \mathrm{mmol} / 1$, respectively. The apoptosis rate was $0.018,0.028,0.033,0.043,0.063$ and 0.079 in the 5.56 to $13.3 \mathrm{mmol} / 1$ glucose + bupivacaine groups, respectively. There were statistically significant differences between the two groups.

Western blot analysis. GRP78 is located in the ER and ER stress may result in the upregulation of GRP78 expression. GRP78 protein expression is shown in Fig. 4. There were statistically significant differences between the glucose and glucose + bupivacaine groups at glucose concentrations of $7.0 \mathrm{mmol} / 1,7.8 \mathrm{mmol} / 1,11.1 \mathrm{mmol} / 1,13.3 \mathrm{mmol} / 1(\mathrm{P}<0.001)$. However, no statistically significant differences were identified between the two groups at glucose concentrations of $5.56 \mathrm{mmol} / 1$ and $6.1 \mathrm{mmol} / 1$ ( $\mathrm{P}>0.05)$.

\section{Discussion}

The incidence of neurological complications in pregnant females with GDM following intraspinal anesthesia is higher than in pregnant females with normal blood sugar levels (1). The risk of neuronal injury is significantly increased in the former case. The complication may be accompanied by an

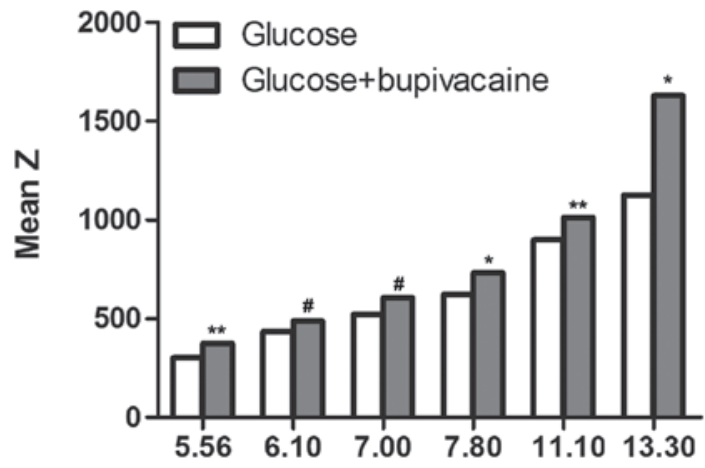

Figure 1. Reactive oxygen species (ROS) production in cells. Levels of ROS were enhanced with increasing glucose concentration. ${ }^{\#} \mathrm{P}>0.05,{ }^{*} \mathrm{P}<0.05$ and ${ }^{* *} \mathrm{P}<0.01$ vs. the glucose group.

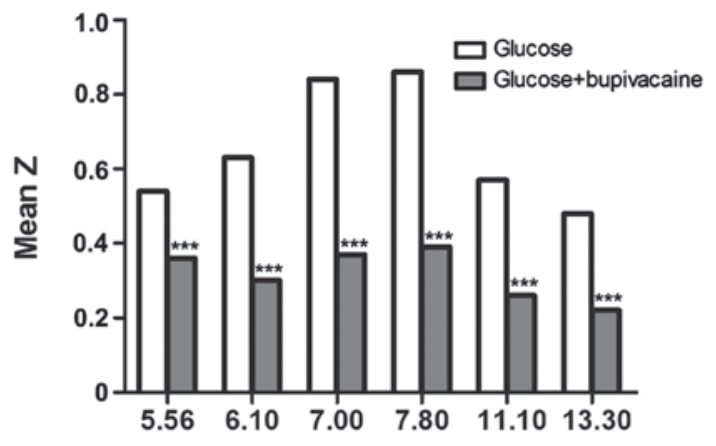

Figure 2. Cell survival analysis. Cell viability was assessed in an MTT assay. The cell viability was enhanced with increasing glucose concentration. ${ }^{* * * *} \mathrm{P}<0.001$ vs. the glucose group.

enhanced susceptibility, such as the aggravation of existing polyneuropathy. Thus, GDM is gaining increasing focus among anesthesiologists. Transient neurologic symptoms (TNS) are the most common clinical symptoms, marked by pain in the lower limbs, hyposthesia or allergy. Occasionally, this is accompanied by weakness in the lower limbs or urinary retention $(14,15)$. Numerous studies have been conducted in regard to peripheral neurotoxicity. In vitro studies are usually performed with SH-SY5Y cells $(16,17)$. As a substrain of the SH-SY cell line, SH-SY5Y is derived from a malignant cancer of the human neural crest. SH-SY5Y cells usually feature a small, round cell body with protruding short axons, a small quantity of cytoplasm and few dendritic processes. They grow in culture flasks in a cluster-like manner. SH-SY5Y cells are similar to normal neuronal cells with respect to morphology, physiology and biochemical function $(18,19)$. Therefore, they are frequently used in in vitro experiments investigating the peripheral neurotoxicity of local anesthetic agents.

At present, topics including changes in the ultrastructure, metabolism and electrophysiology of neuronal tissues in patients with GDM as a result of the use of local anesthetic agents have evoked particular discussion. In addition, efforts have been made to reduce such damages caused by local anesthetic agents. GDM is a specific type of diabetes diagnosed in pregnant females, which is unlike type I and II diabetes mellitus or increased blood sugar levels. In females with GDM, the oxidation and stress levels are usually increased. 

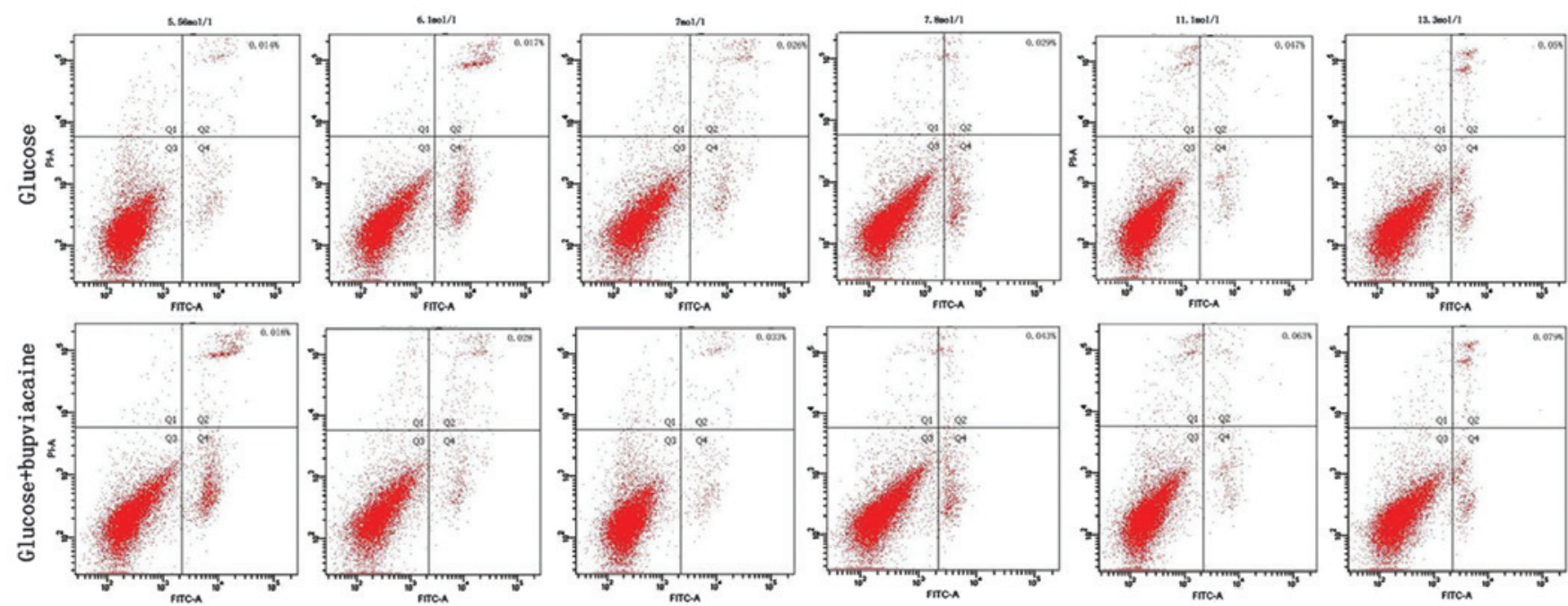

Figure 3. Flow cytometry (FCM) detection of apoptosis. The apoptosis rate was $0.014,0.017,0.026,0.029,0.047$ and 0.05 in the 5.56 to 13.3 mmol/1 glucose groups, respectively. Bn comparison, the apoptosis rate was $0.018,0.028,0.033,0.043,0.063$ and 0.079 in the 5.56 to $13.3 \mathrm{mmol} / 1$ glucose + bupivacaine groups, respectively. FITC, fluorescein isothiocyanate; PI, propidium iodide.
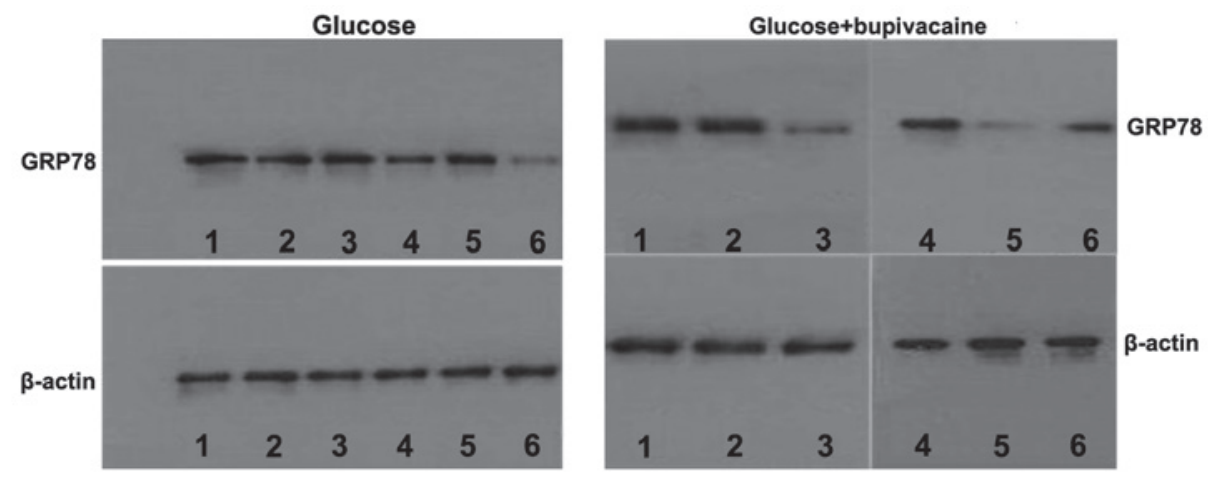

Figure 4. Western blot analysis of the $78 \mathrm{kDa}$ glucose-regulated protein (GRP78). There were statistically significant differences between the two groups (glucose and glucose+bupivacaine) at glucose concentrations of $7.0 \mathrm{mmol} / 1(\mathrm{P}<0.001), 7.8 \mathrm{mmol} / 1(\mathrm{P}<0.001), 11.1 \mathrm{mmol} / 1(\mathrm{P}<0.001)$ and $13.3 \mathrm{mmol} / 1(\mathrm{P}=0.001)$; however, no statistically significant differences were observed between the two groups at glucose concentrations of $5.56 \mathrm{mmol} / 1$ (P>0.05) and $6.1 \mathrm{mmol} / 1$ (P>0.05). Lanes: 1, $5.56 \mathrm{mmol} / \mathrm{l} ; 2,6.1 \mathrm{mmol} / 1 ; 3,7.0 \mathrm{mmol} / 1 ; 4,7.8 \mathrm{mmol} / \mathrm{l} ; 5,11.1 \mathrm{mmol} / 1 ; 6,13.3 \mathrm{mmol} / 1$.

ER stress is associated with the metabolic disorder in diabetes mellitus. It has been suggested that pregnancy may result in an enhanced susceptibility of neuronal tissue to local anesthetic agents. However, little is known regarding the mechanism of how local anesthetic agents damage the spinal neuronal system.

The changes in expression of GRP78 in the ER were assessed in this study. When the cells were treated with glucose at low levels to induce the expression of GRP78, the homeostasis of the ER was able to be maintained. The upregulation of GRP expression may be used as an indicator of ER stress (20). With an increasing glucose concentration, ER stress is enhanced. As a result, the oxidative stress increases, causing the toxic accumulation of ROS in cells, and apoptosis may occur. In response to this, GRP78 expression begins to decline. As the glucose concentration continues to increase, the synthesis of large quantities of GRP78 is triggered due to enhanced ER stress, which activates a self-repair mechanism. At this time, GRP78 expression is upregulated. However, an excessively high glucose concentration ultimately destroys the homeostasis of the ER under stress. In response to this, apoptosis is initiated, so that a reduced quantity of GRP78 is able to be produced. In the present study, when the glucose concentration was $13.3 \mathrm{mmol} / \mathrm{l}$, the production of GRP78 increased again following treatment with bupivacaine. This may have been a result of the self-repair mechanism of the cells. This finding appears to indicate the existence of a biphasic effect of the unfolded protein response (UPR) on cell survival (21-24). The expression of GRP78 is negatively correlated with the number of apoptotic cells. GRP78 has a crucial role in evading apoptosis caused by ER stress. The upregulated expression of GRP78 is capable of alleviating the damage of ER stress to cells, as GRP78 exhibits anti-apoptotic activity. However, as the toxic accumulation of ROS continues, the protective effect of GRP78 appears to be inadequate.

In the present study, prior to the use of the local anesthetic agent, ROS production and the levels of apoptosis were enhanced with increasing blood sugar levels in SH-SY5Y cells. The differences between the groups incubated at different glucose concentrations exhibited statistical significance. 
This suggests that ER stress is enhanced at increased blood sugar levels, triggering an upregulated expression of GRP78 to maintain the homeostasis of the ER. As blood sugar levels continue to increase, ER stress is further enhanced, resulting in higher levels of oxidative stress. ROS accumulate in cells and cause apoptosis. Thus, the expression of GRP78 is ultimately reduced. The cells, which are no longer able to maintain the homeostasis of the ER, have no other fate but apoptosis. As a result, the production of GRP78 is significantly reduced.

Changes in GRP78 expression were observed in the ER of SH-SY5Y cells following treatment with bupivacaine for $6 \mathrm{~h}$. It was demonstrated that at lower glucose levels, homeostasis was effective to sustain the cell functions. However, as the blood sugar levels continued to increase, enhanced ER stress resulted in the increase of oxidative stress. ROS accumulation in cells ultimately led to apoptosis and a reduction in the expression of GRP78.

Following bupivacaine treatment for $6 \mathrm{~h}$, significant differences were observed in ROS production and the level of apoptosis in comparison with the glucose group. It may be concluded that bupivacaine significantly promotes ER stress in SH-SY5Y cells to sustain homeostasis. When the ER stress continues to increase, the oxidative stress increases correspondingly, and more ROS accumulate. The same pattern was observed in the changes in the levels of GRP78 expression (statistically significant) and apoptosis (statistically significant), as indicated by the results of the glucose and glucose + bupivacaine groups.

Following bupivacaine treatment, the levels of ROS generation and apoptosis increased with increasing blood sugar levels, and the difference was statistically significant. Therefore, the effect of high blood sugar levels on ER stress and apoptosis in SH-SY5Y cells even persisted following bupivacaine treatment. The combined effect of bupivacaine and high glucose levels exceeded that of either alone with regard to the levels of ROS generation and apoptosis. Thus, the interaction between bupivacaine and glucose contributes to the enhancement of ER stress and apoptosis. The changes in ROS production observed in this study demonstrate that the energy and nutrient surplus trigger ER stress. The stress on organelles and the mitochondrial dysfunction promotes further production of ROS, which, in turn, completes the cycle, as ER stress is further increased. The increased ER stress results in oxidative stress, and ROS accumulation in the cells, leading to toxicity. This persistent ER stress facilitates apoptosis and high blood sugar levels are the trigger of this cycle. The increase in ROS production following bupivacaine treatment indicates that bupivacaine is another trigger of the cycle. When SH-SY5Y cells were exposed to the two factors (bupivacaine and high blood sugar levels) the levels of ROS production and apoptosis were significantly higher than in the case of only one factor being present. ROS production was markedly increased, leading to higher neurotoxicity and higher levels of apoptosis. The combined effects of the two factors further aggravated ER stress and the disturbance of the internal environment, as well as enhancing apoptosis.

It may be concluded that under the presence of a glucose stimulus, ER functions are altered in SH-SY5Y cells, causing ER stress. At lower glucose concentrations, cells are able to maintain the stability of the internal environment of the ER through the upregulated expression of GRP78 due to the action of homeostasis. ER stress is enhanced with increasing glucose concentration and the stress is aggravated when combined with the use of anesthetic agents. The resulting increase in ROS production leads to enhanced cytotoxicity. When the protective effect of GRP78 is not able to further counteract the large quantities of ROS or remove the ROS, their cytotoxic effect causes the initiation of apoptosis. The induction of GRP78 transcription is important for preventing apoptosis in response to ER stress in cells $(25,26)$. Once the response to ER stress is triggered, the expression of GRP78 is upregulated to achieve the optimum level of damage protection of the ER. In this case, the cells are able to adapt to the stress and survive. Following the peak period of stress, GRP78 expression is reduced. However, with the persistent stress, the damage is aggravated and dysfunction of the ER occurs. At this time, ER stress is no longer able to be alleviated through the upregulation of GRP78 expression, and therefore apoptosis is activated. It is indicated that normal glucose levels are the optimum conditions for the growth of neuronal cells and the recovery from toxicity damage caused by local anesthetic agents.

The limitation of this study lies in the fact that no primary SH-SY5Y cells were used for the in vitro study, so the situation may deviate from clinical circumstances.

The present study shows that ER stress induced by high glucose levels in neuronal cells is associated with apoptosis. Inducing the expression of GRP78 may alleviate neuronal cell damage and apoptosis, and exert a protective effect on neuronal cells. Due to the fact that the mechanism of ER stress is associated with the apoptosis of neuronal cells at high glucose levels, GRP78 may become a candidate target site. Inducing the expression of GRP78 is a potential means of therapy for treating the enhanced susceptibility to neurotoxicity caused by local anesthetic agents in patients with GDM.

In conclusion, the present study reveals the role of ROS in the susceptibility to neurotoxicity of local anesthetic agents in a model of GDM and provides an experimental basis for its treatment and control.

\section{Acknowledgements}

The authors would like to thank the open platform of medical experimental technologies of the Teaching and Research Department of Cytobiology, Preclinical Medical College, Southern Medical University (Guangzhou, China) for providing the experimental facilities and technical support. This study was supported by a grant from the Science and Technology Development Program of Shenzhen (project no. 201203089)

\section{References}

1. Arakawa M: Does pregnancy increase the efficacy of lumbar epidural anesthesia? Int J Obstet Anesth 13: 86-90, 2004.

2. Kühl C: Insulin secretion and insulin resistence in pregnancy and GDM. Implications for diagnosis and management. Diabetes 40 (Suppl 2): 18-24, 1991

3. Zhang C, Schulze MB, Solomon CG and Hu FB: A prospective study of dietary patterns, meat intake and the risk of gestational diabetes mellitus. Diabetologia 49: 2604-2613, 2006.

4. Zhang F, Dong L, Zhang CP et al: Increasing prevalence of gestational diabetes mellitus in Chinese women from 1999 to 2008. Diabet Med 28: 652-657, 2011.

5. Engin F and Hotamisligil GS: Restoring endoplasmic reticulum function by chemical chaperones: an emerging therapeutic approach for metabolic diseases. Diabetes Obes Metab 12: 108-115, 2010 
6. Saldana TM, Siega-Riz AM and Adair LS: Effect of macronutrient intake on the development of glucose intolerance during pregnancy. Am J Clin Nutr 79: 479-486, 2004.

7. Ying $\mathrm{H}$ and Wang DF: Effects of dietary fat on onset of gestational diabetes mellitus. Zhonghua Fu Chan Ke Za Zhi 41: 729-731, 2006 (In Chinese).

8. Hotamisligil GS: Endoplasmic reticulum stress and the inflammatory basis of metabolic disease. Cell 140: 900-917, 2010.

9. Park CJ, Park SA, Yoon TG, et al: Bupivacaine induces apoptosis via ROS in the Schwann cell line. J Dent Res 84: 852-857, 2005.

10. Zhou SQ, Lu J, Zhang QG, et al: Is activation of AMP-activated protein kinase involved in ropivacaine-induced SH-SY5Y cell apoptosis? Chin J Anesthesiol 31: 197-200, 2011 (In Chinese).

11. Rao RV, Ellerby HM and Bredesen DE: Coupling endoplasmic reticulum stress to the cell death program. Cell Death Differ 11: 372-380, 2004.

12. Zorov DB, Juhaszova M and Sollott SJ: Mitochondrial ROS-induced ROS release: an update and review. Biochim Biophys Acta 1757: 509-517, 2006.

13. Lin JH, Li H, Yasumura D, et al: IRE1 signaling affects cell fate during the unfolded protein response. Science 318: 944-949, 2007.

14. Rorarius M, Suominen P, Haanpaa M, et al: Neurologic sequelae after caesarean section. Acta Anaesthesiol Scand 45: 34-41, 2001.

15. Auroy Y, Benhamou D, Bargues L, et al: Major complications of regional anesthesia in France: The SOS Regional Anesthesia Hotline Service. Anesthesiology 97: 1274-1280, 2002.

16. Werdehausen R, Fazeli S, Braun S, et al: Apoptosis induction by different local anaesthetics in a neuroblastoma cell line. Br J Anaesth 103: 711-718, 2009.

17. Donzelli E, Carfì M, Miloso M et al: Neurotoxicity of platinum compounds: comparison of the effects of cisplatin and oxaliplatin on the human neuroblastoma cell line SH-SY5Y. J Neurooncol 67: 65-73, 2004.
18. Biedler JL, Roffler-Tarlov S, Schachner M and Freedman LS: Multiple neurotransmitter synthesis by human neuroblastoma cell lines and clones. Cancer Res 38: 3751-3757, 1978.

19. Raghunath M, Patti R, Bannerman P, et al: A novel kinase, AATYK induces and promotes neuronal differentiation in a human neuroblastoma (SH-SY5Y) cell line. Brain Res Mol Brain Res 77: 151-162, 2000.

20. Blagosklonny MV: Cell death beyond apoptosis. Leukemia 14: 1502-1508, 2000.

21. Hollien J, Lin JH, Li H, et al: Regulated Irel-dependent decay of messenger RNAs in mammalian cells. J Cell Biol 186: 323-331, 2009.

22. Hollien J and Weissman JS: Decay of endoplasmic reticulum-localized mRNAs during the unfolded protein response. Science 313: 104-107, 2006.

23. Oikawa D, Kimata Y, Kohno K and Iwawaki T: Activation of mammalian IRE1alpha upon ER stress depends on dissociation of $\mathrm{BiP}$ rather than on direct interaction with unfolded proteins. Exp Cell Res 315: 2496-2504, 2009.

24. Ron D and Walter P: Signal integration in the endoplasmic reticulum unfolded protein response. Nat Rev Mol Cell Biol 8: 519-529, 2007.

25. Walter L and Hajnóczky G: Mitochondria and endoplasmic reticulum: the lethal interorganelle cross-talk. J Bioenerg Biomembr 37: 191-206, 2005.

26. Mattson MP: Neuronal life-and-death signaling, apoptosis, and neurodegenerative disorders. Antioxid Redox Signal 8: 1997-2006, 2006. 